\title{
On Enhancing the Performance of the DPTE-Based Noise Cancellation Method Utilizing the PTS PAPR Reduction Scheme in PLC Systems
}

\author{
Khaled M. Rabie and Emad Alsusa \\ School of Electrical and Electronic Engineering, \\ The University of Manchester, United Kingdom, M13 9PL, \\ Emails: \{khaled.rabie@manchester.ac.uk; e.alsusa@manchester.ac.uk\}
}

\begin{abstract}
Several methods have been proposed in the literature for impulsive noise (IN) blanking and the most efficient of which is the dynamic peak-based threshold estimation (DPTE). In this method only estimates of the signal peaks are required in order to optimally blank the noise. In this paper we propose to enhance the capability of the conventional DPTE technique by pre-processing the OFDM signal at the transmitter. This is simply done by applying the partial transmit sequence (PTS) scheme. To evaluate system performance we consider the probability of blanking error $\left(P_{b}\right)$, probability of missed blanking $\left(P_{m}\right)$, probability of successful detection $\left(P_{s}\right)$ and the signal-to-noise ratio (SNR) at the output of the blanking device. The results reveal that DPTE-PTS technique can significantly minimize both $P_{b}$ and $P_{m}$ and maximize $P_{s}$. Furthermore, it will be shown that the proposed system can achieve up to $1.5 \mathrm{~dB}$ SNR enhancement over the conventional DPTE scheme.
\end{abstract}

Index Terms-Impulsive noise, partial transmit sequence (PTS), peak-to-average power ratio (PAPR), power-line communications (PLC), smart grid, signal-to-noise ratio (SNR).

\section{INTRODUCTION}

$\mathbf{S}$ MART grids can be realized with a heterogeneous set of networks such as Wi-Fi, coaxial cables, fiber optics, power-line networks etc [1]. The fact that power-line networks are already in existence makes this technology more attractive for smart grid developers to retrofit such networks for communications. However, this requires overcoming many challenges including the impulsive noise (IN), frequency-dependent attenuation, multipath fading and electromagnetic compatibility (EMC) issues. In particular, IN is the main concern and is the most dominant factor responsible for degrading communication signals. In order to evaluate system performance in IN environments an accurate noise model is needed. The two-component mixture-Gaussian noise model, [2], [3], has been the most commonly used in evaluating the system performance in such environment and therefore will be adopted in this work.

A number of methods with different degrees of complexity have been reported in the literature to improve the performance of orthogonal frequency division multiplexing (OFDM) based receivers in IN channels [4], [5]. The simplest of such methods is to precede the OFDM demodulator with blanking device to zero the received signal when it exceeds a certain threshold [6], [7]. Theoretical performance analysis to find closed-form expressions for the signal-to-noise ratio (SNR) at the output of the blanking device first appeared in [3], [8]. The main disadvantage of this method, however, is that in order to optimally suppress IN, the noise characteristics must be accurately known apriori. In this paper we refer to this method as the unmodified method. It is shown in [9] that even for small error estimations of the IN, the unmodified method can suffer from significant performance degradation. Furthermore, the authors introduced a different criterion for estimating the optimal blanking threshold (OBT) independently of the IN parameters by using estimates of the transmitted signals' peak to average power ratio (PAPR); this method is implemented in [10] and was referred to as dynamic peak-based threshold estimation (DPTE). This technique not only completely eliminates the need for prior knowledge about the characteristics of IN but can also achieve a gain of up to $2.5 \mathrm{~dB}$ over the unmodified method if the signal peaks can be estimated accurately.

Imperfect recognition of the IN signal may lead to nulling uncorrupted or overlooking corrupted signals which can result in high probability of blanking error and high probability of missed detection, respectively and, hence, dramatic performance deterioration. In this paper we proposed to enhance the capability of the conventional DPTE technique by preprocessing the OFDM signal at the transmitter. This is simply done by applying the well-known PAPR reduction scheme, namely, partial transmit sequence (PTS) [11]. Such technique is referred to here as DPTEPTS. Processing the OFDM signal in a such way in combination with applying the DPTE technique is able to minimize the probability of blanking error $\left(P_{b}\right)$ and probability of missed detection $\left(P_{m}\right)$ while improving the probability of successful detection $\left(P_{s}\right)$. Therefore, these probabilities are investigated for the unmodified, conventional DPTE and DPTE-PTS techniques. In addition and for more quantitative characterization of the system performance, the SNR at the output of the blanker is also considered. It is found that that the DPTE-PTS technique is able to reduce $P_{b}$ and $P_{m}$ considerably as well as improving $P_{s}$. It is also shown that the proposed system can attain up to $3.5 \mathrm{~dB}$ and 1.5 dB SNR gains relative to the unmodified method and the conventional DPTE scheme, respectively.

The rest of the paper is organized as follows. In Section II, the system model is presented. In Section III, the complementary cumulative distribution function (CCDF) is discussed for the unmodified and OFDM-PTS systems. The probability of blanking error, probability of missed blanking and probability of successful detection are assessed for the unmodified, conventional DPTE and DPTE-PTS systems in Section IV, V and VI, respectively. Section VII discusses the simulation results for the output SNR performance. Finally conclusions are drawn in Section VIII. 


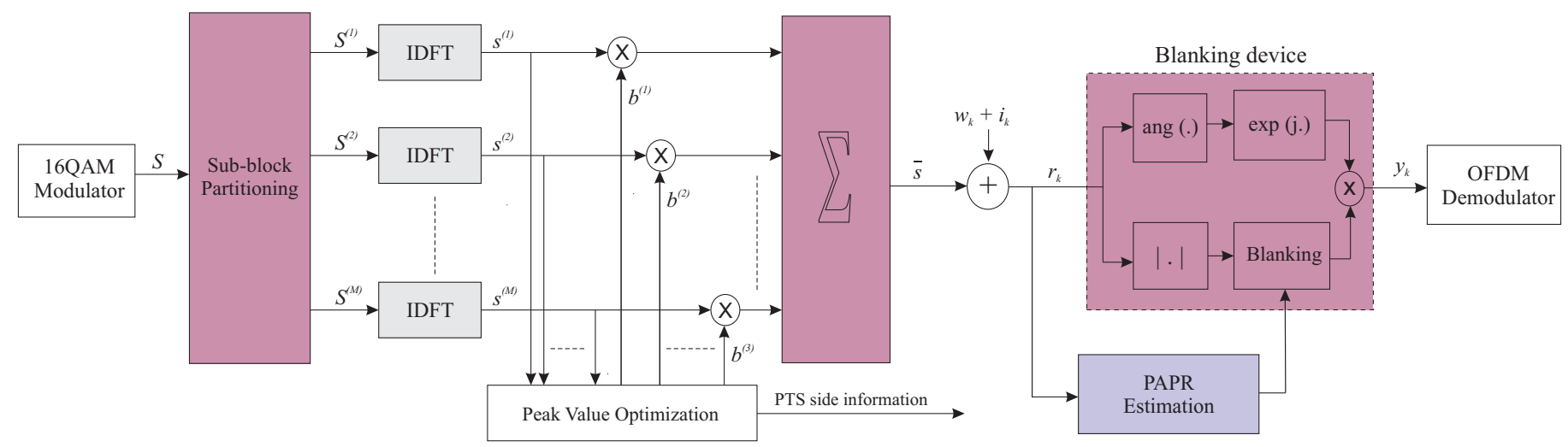

Figure 1: Block diagram of OFDM system with PTS-based PAPR reduction at the transmitter and blanking at the receiver.

\section{System Model}

Fig. 1 illustrates the system diagram of this study. The information bits are first mapped into 16QAM symbols $(S)$ and each block of these symbols is then partitioned into $M$ disjoint sub-blocks of length $N$ to produce $S^{(m)}=$ $\left[S_{0}^{(m)}, S_{1}^{(m)}, \ldots, S_{N-1}^{(m)}\right], \quad m=1,2, \ldots, M$. The subcarriers positions in $S^{(m)}$ which are presented in another sub-block are set to zero so that $S=\sum_{m=1}^{M} S^{(m)}$. Then inverse fast Fourier transform (IFFT) is employed for each sub-block to produce $s^{(m)}=\operatorname{IFFT}\left\{S^{(m)}\right\}$. After that each sub-block is multiplied by a different phase weighting factor $b^{(m)}$. The peak value optimization block iteratively searches for the optimal combination of the phase weighting factors that offers the minimum PAPR. Once the optimal weighting factor is determined, all the subblocks are summed as $\tilde{s}=\sum_{m=1}^{M} b^{(m)} s^{(m)}$ and then transmitted. In generally, the PAPR of the OFDM signal is defined as

$$
\text { PAPR }=\frac{\max |\tilde{s}(t)|^{2}}{\mathbb{E}\left[|\tilde{s}(t)|^{2}\right]}, \quad 0<t<T_{s}
$$

where $\mathbb{E}[$.$] is the expectation function. When \tilde{s}(t)$ is passed through the IN channel, the received signal becomes

$$
r_{k}=\tilde{s}+n_{k}, \quad k=0,1,2, \ldots, N-1
$$

In this paper, the two-component mixture-Gaussian noise model is adopted in which IN is modeled as a Bernoulli-Gaussian random process [2] and is given by

$$
n_{k}=w_{k}+i_{k}, \quad k=0,1,2, \ldots, N-1
$$

where

$$
i_{k}=b_{k} g_{k}, \quad k=0,1,2, \ldots, N-1
$$

$\tilde{s}_{k}, w_{k}$ and $i_{k}$ are assumed to be mutually independent. $n_{k}$ is the total noise component, $w_{k}$ is the additive white Gaussian noise (AWGN), $i_{k}$ is the IN, $g_{k}$ is complex white Gaussian noise with mean zero and $b_{k}$ is the Bernoulli process with probability $\operatorname{Pr}\left(b_{k}=1\right)=p$, where $p$ denotes the probability occurrence of IN. The probability density function (PDF) of the total noise can be expressed as

$$
P_{n_{k}}\left(n_{k}\right)=(1-p) \mathcal{G}\left(n_{k}, 0, \sigma_{w}^{2}\right)+p \mathcal{G}\left(n_{k}, 0, \sigma_{w}^{2}+\sigma_{i}^{2}\right)
$$

where $\mathcal{G}($.$) is the Gaussian PDF given by \mathcal{G}\left(x, \mu, \sigma_{x}^{2}\right)=$ $\frac{1}{\sqrt{2 \pi \sigma_{x}^{2}}} e^{-\frac{(x-\mu)^{2}}{2 \sigma_{x}^{2}}}, \sigma_{w}^{2}$ and $\sigma_{i}^{2}$ are the AWGN and IN variances which are related to the input SNR and signal-to-impulsive noise ratio $(\mathrm{SINR})$ as $\mathrm{SNR}=10 \log _{10}\left(\frac{\sigma_{s}^{2}}{\sigma_{w}^{2}}\right)$ and SINR $=$ $10 \log _{10}\left(\frac{\sigma_{s}^{2}}{\sigma_{i}^{2}}\right)$, respectively. At the receiver front-end, blanking is performed and depending on the scenario we have three different systems.

\section{- Unmodified Method:}

In this method PTS is not applied and only one conventional OFDM modulator is used. At the receiver conventional blanking is applied as

$$
y_{k}=\left\{\begin{array}{ll}
r_{k}, & \left|r_{k}\right| \leq T \\
0, & \left|r_{k}\right|>T
\end{array} \quad k=0,1, \ldots, N-1\right.
$$

where $T$ is the blanking threshold, $r_{k}$ and $y_{k}$ are the input and output of the blanker, respectively. It is important to stress that determining the OBT in this method requires accurate knowledge about the characteristics of IN which may not be easily obtainable in practice. A theoretical expression for the OBT $\left(T_{\text {opt }}\right)$ was derived as a function of IN parameters by Zhidkov [8, Eq. (28)] as well as the output SNR given as

$$
\mathrm{SNR}_{\text {unmod. }}=\frac{2}{\mathbb{E}\left[A_{n}^{2}\right]}
$$

where $\mathbb{E}\left[A_{n}^{2}\right]$ is defined as in [8, Eq. (26)]. These expressions will be used to provide a comparative analysis to show the superiority of the proposed system and also to verify the accuracy of our simulation model.

\section{- Conventional DPTE Method:}

In this system, blanking is performed based on the OFDM symbol peak estimates irrespective of IN characteristics when a conventional OFDM modulator is used at the transmitter and 


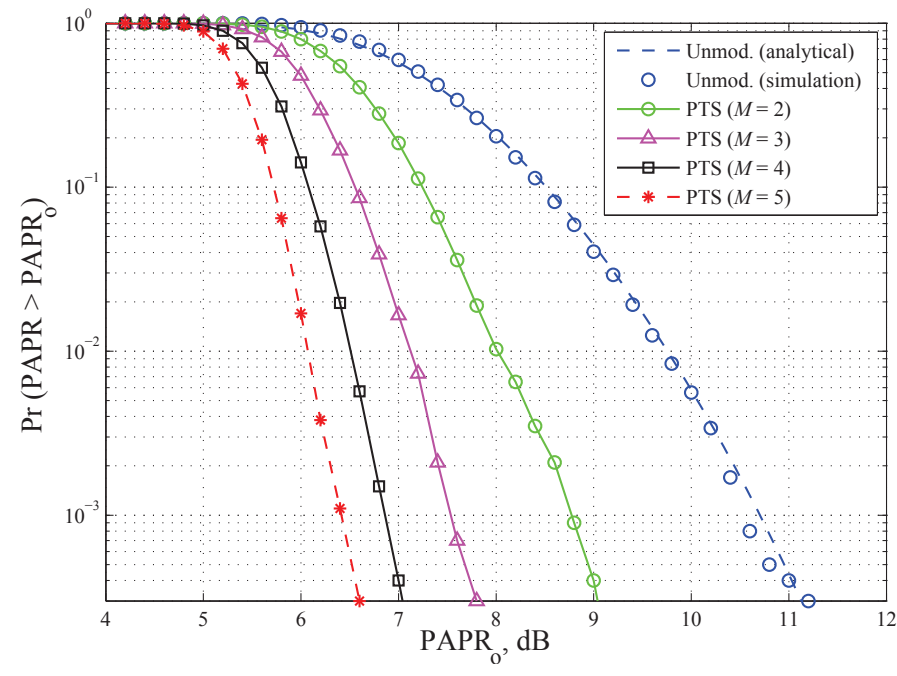

Figure 2: CCDF plot of PTS for different sub-block sizes when $W=4$.

its principle is

$$
y_{k}=\left\{\begin{array}{ll}
r_{k}, & \left|r_{k}\right| \leq \tilde{P} \\
0, & \left|r_{k}\right|>\tilde{P}
\end{array} \quad k=0,1, \ldots, N-1\right.
$$

where $\tilde{P}$ is the estimated OFDM symbol peak.

- DPTE-PTS Method:

This method is similar to the conventional one but with applying a PTS modulator at the transmitter, i.e. when signal peak is reduced. The PAPR reduction is usually measured in terms of the complementary cumulative distribution function (CCDF).

\section{Complementary Cumulative Distribution FUNCTION (CCDF)}

The CCDF of PAPR is defined as the probability that the PAPR of a data block exceeds a given threshold $\left(\mathrm{PAPR}_{o}\right)$ and is expressed as

$$
C C D F=1-\operatorname{Pr}\left(P A P R \leq P A P R_{o}\right)
$$

A simple expression of the CCDF for the unmodified system is derived in [12] as

$$
C C D F=1-\left(1-e^{\left(-P A P R_{o}\right)}\right)^{N}
$$

In the unmodified system only one IFFT operation is required whereas in the PTS scheme $M$ IFFT operations are performed. In the latter scheme a set of phase weighting factors is usually selected for generating the phase weighting sequences. Assuming that there are $W$ phase weighting factors in this set, the optimal PAPR is found after checking $W^{M-1}$ different combinations and the number of bits required to represent the side information is $\log _{2}\left(W^{M-1}\right)$. The amount of PAPR reduction for this scheme depends on both the number of partitions $(M)$ and the number of phase weighting sequences $(W)$.

Fig. 2 depicts the CCDF of the PAPR for different numbers of sub-blocks $(M=1,2,3,4,5)$. In this investigation, the phase weighting factors are chosen from $W=\{ \pm 1, \pm j\}$ as it is found

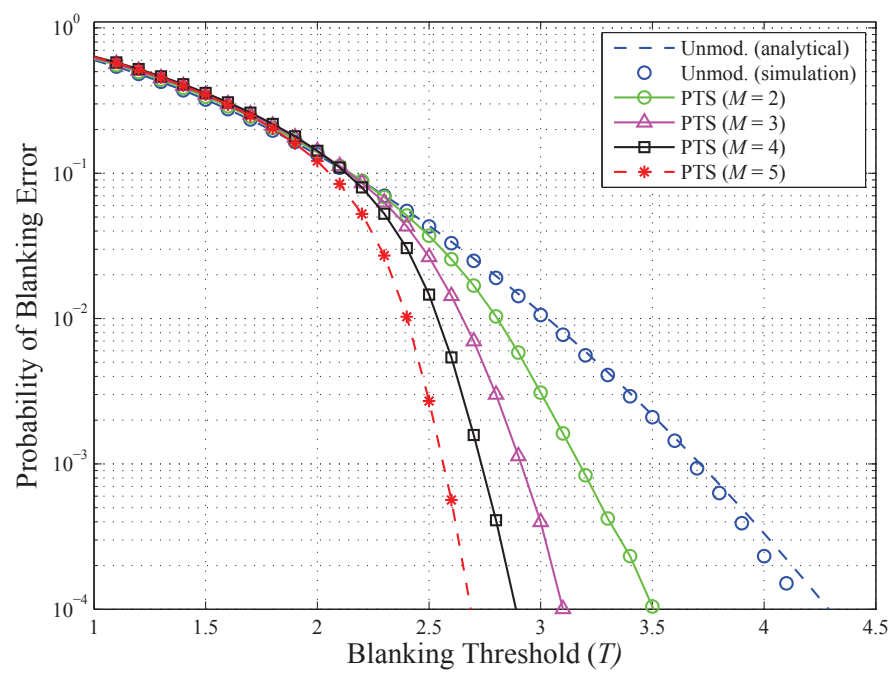

Figure 3: Probability of blanking error versus blanking threshold for different sub-block sizes when $W=4$.

in [11] that a restriction to four weighting phase factors can provide a significant peak reduction. It is evident from this figure that the amount of PAPR reduction increases as the number of partitions is increased and this improvement becomes less significant for large values of $M$. The PAPR reduction implies that more of the transmitted signal energy is contained close to the average value and hence IN becomes more distinguishable at the receiver resulting in a more efficient IN reduction process.

\section{Probability of Blanking ERror}

The probability of blanking error $\left(P_{b}\right)$ is the probability that the amplitude of the received sample, $A_{r}=\left|r_{k}\right|$, exceeds the blanking threshold when it is unaffected by IN. $P_{b}$ is defined by the joint probability $P\left(B, \mathcal{H}_{0}\right)$, where $B$ is the event of blanking the received signal exceeding $T$ and the null hypothesis $\mathcal{H}_{0}$ implies the absence of IN, $P\left(\mathcal{H}_{0}\right)=(1-p)$. $P_{b}$ is expressed as

$$
P_{b}=P\left(A_{r}>T \mid \mathcal{H}_{0}\right) P\left(\mathcal{H}_{0}\right)
$$

In the absence of IN, the amplitude of the unmodified received signal has Rayleigh distribution with parameter $\sigma^{2}=\sigma_{s}^{2}+\sigma_{w}^{2}$. Therefore, it is found in [13] that

$$
P_{b}^{\text {unmod }}=\exp \left(-\frac{T^{2}}{2\left(\sigma_{s}^{2}+\sigma_{w}^{2}\right)}\right)(1-p)
$$

In the case of the OFDM-PTS system, $P_{b}$ is found by means of simulation. Our simulations from this point onward are based on an OFDM system consisting of $N=64$ subcarriers with 16QAM modulation and the OFDM signal power is normalized as $\sigma_{s}^{2}=(1 / 2) E\left[\left|s_{k}\right|^{2}\right]=1$. Fig. 3 illustrates the $P_{b}$ for the unmodified and PTS-based systems with input SNR $=40 \mathrm{~dB}$ for various values of $M$. For the unmodified system it is obvious that the analytical and simulated results are in good agreement. It is also clear that the behavior of the probability can be divided into two regions. The first region is when $\{T \lesssim 2\}$ during which the PTS-based scheme does not provide any probability reduction in comparison with that of the unmodified method. In the second 


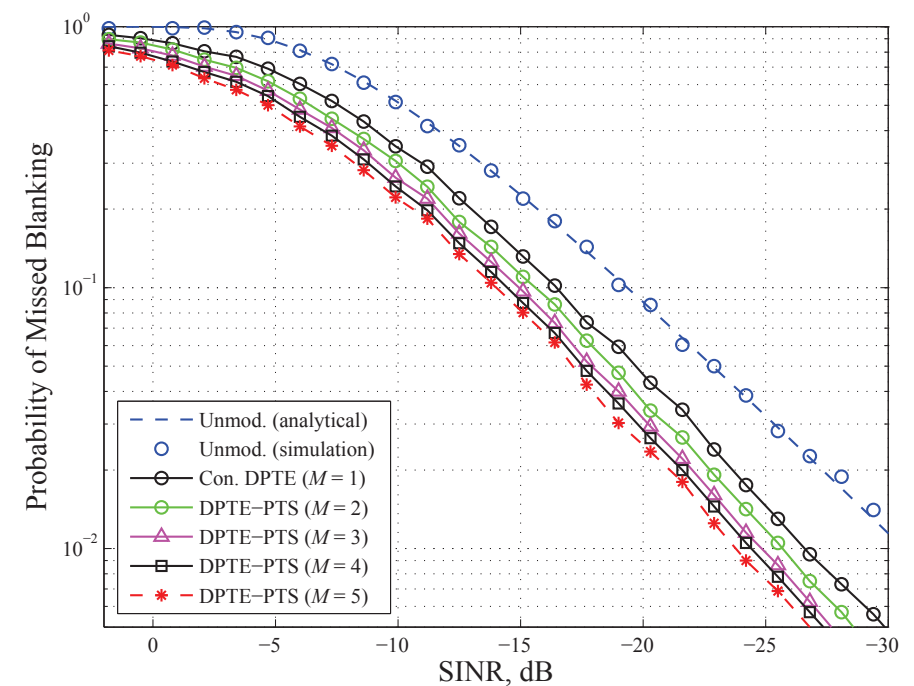

Figure 4: Probability of missed blanking versus SINR for the unmodified, conventional DPTE and DPTE-PTS techniques with various values of $M$ when $W=4$.

region $\{T>2\}$, however, it is noticeable that the proposed technique minimizes $P_{b}$ compared to the unmodified method and that this probability is inversely proportional to $M$ and $T$. For instance when $\{M=5\}$ and at $T=2.5$, the probability is reduced by about 1.5 order of magnitude whereas for $T=2.75$, the probability is minimized by about 2.5 orders of magnitude. This suggests that the system performance will improve for higher values of $M$ as will be further discussed later.

However, after the OFDM signal is passed through the IN channel two other important measures of the system performance, which highly depend on the IN characteristics, should be used instead of $P_{b}$, namely, $P_{m}$ and $P_{s}$ both of which are discussed below.

\section{Probability of Missed Blanking}

$P_{m}$ is defined as the probability that the affected signals are not blanked and is given by the joint probability $P\left(\bar{B}, \mathcal{H}_{1}\right)$ where $\bar{B}$ denotes the absence of blanking and the alternative hypothesis $\mathcal{H}_{1}$ implies the presence of IN, $P\left(\mathcal{H}_{1}\right)=p$. In this section we investigate $P_{m}$ for the unmodified and DPTE methods.

\section{A. Unmodified Method}

For the unmodified method, $P_{m}$ is expressed as

$$
P_{m}=P\left(A_{r}<T \mid \mathcal{H}_{1}\right) P\left(\mathcal{H}_{1}\right)
$$

Since the amplitude of the unmodified received signal, in the presence of IN, has Rayleigh distribution with parameter $\sigma^{2}=$ $\sigma_{s}^{2}+\sigma_{w}^{2}+\sigma_{i}^{2}$, it is shown that $P_{m}$ for the unmodified scheme is [13]

$$
P_{m}^{\text {unmod }}=\left(1-\exp \left(-\frac{T^{2}}{2\left(\sigma_{s}^{2}+\sigma_{w}^{2}+\sigma_{i}^{2}\right)}\right)\right) p
$$

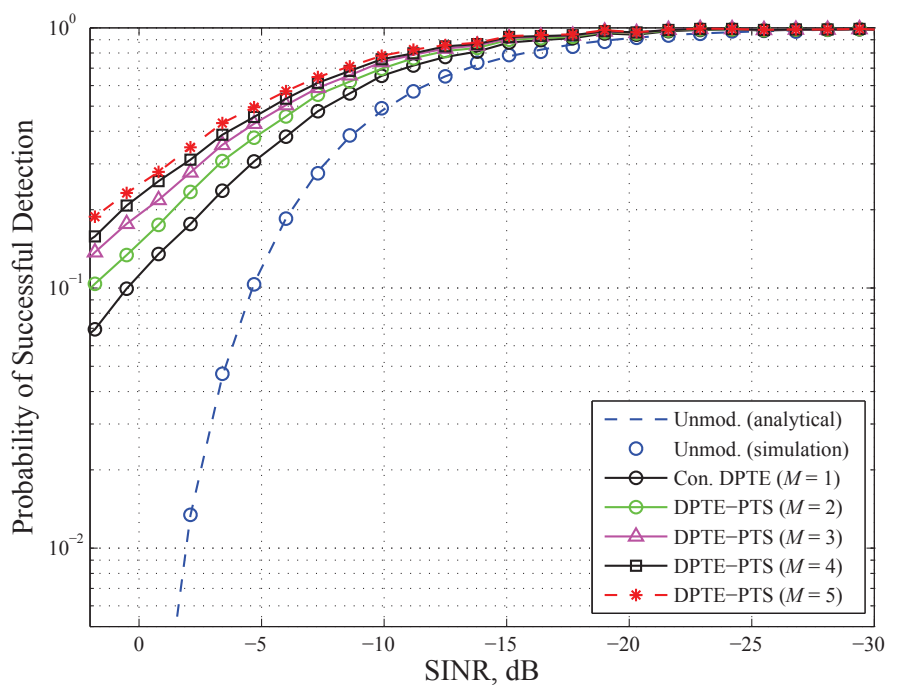

Figure 5: Probability of successful detection versus SINR for the unmodified, conventional DPTE and DPTE-PTS techniques with various values of $M$ when $W=4$.

\section{B. DPTE Method}

For the conventional DPTE and DPTE-PTS schemes, $P_{m}$ is determined as

$$
P_{m}^{D P T E}=P\left(A_{r}<\mathrm{P} \mid \mathcal{H}_{1}\right) P\left(\mathcal{H}_{1}\right)
$$

where $\mathrm{P}$ is the peak value of the associated OFDM symbol.

Fig. 4 depicts some numerical results of (14) as a function of SINR along with simulation results for the unmodified, conventional DPTE and DPTE-PTS systems when SNR $=40 \mathrm{~dB}$. It is important to stress the fact that the results of the unmodified system are obtained under the assumption of perfect IN detection, i.e. substituting $T$ with $T_{o p t}$ [8, Eq. (28)]. It is clear from this figure that the unmodified system has the worst performance and the conventional DPTE system outperforms the unmodified one. We can also note that the DPTE-PTS system offers the best performance and that as $M$ increases the performance improves. In general, it can be observed, for the three systems, that as IN becomes smaller the probability of missed blanking worsens and it improves for very low SINR values. This is justified by the fact that when SINR becomes closer to zero, the amplitude of the OFDM and IN signals become more comparable leading to inaccurate blanking.

\section{Probability of Successful Detection}

$P_{s}$ is another important performance measure after the OFDM signal is passed through the PLC channel and it is defined as the probability of correctly blanking the contaminated samples. $P_{s}$ is given by the joint probability $P\left(B, \mathcal{H}_{1}\right)$ and is discussed below for both the unmodified and DPTE techniques.

\section{A. Unmodified Method}

For the unmodified method, $P_{s}$ is given as

$$
P_{s}=P\left(A_{r}>T \mid \mathcal{H}_{1}\right) P\left(\mathcal{H}_{1}\right)
$$

Hence [13], 


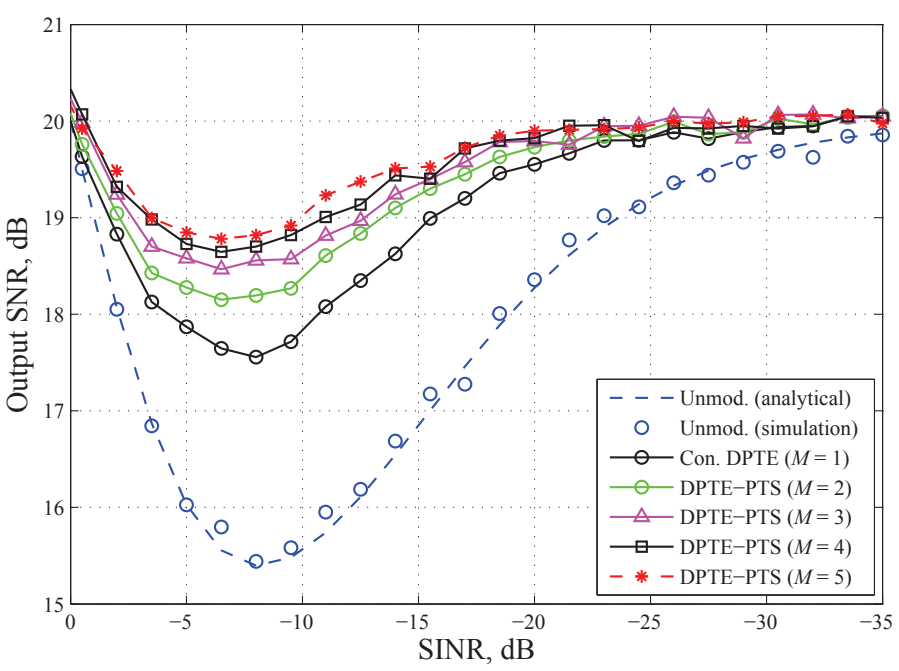

(a) $p=0.01$.

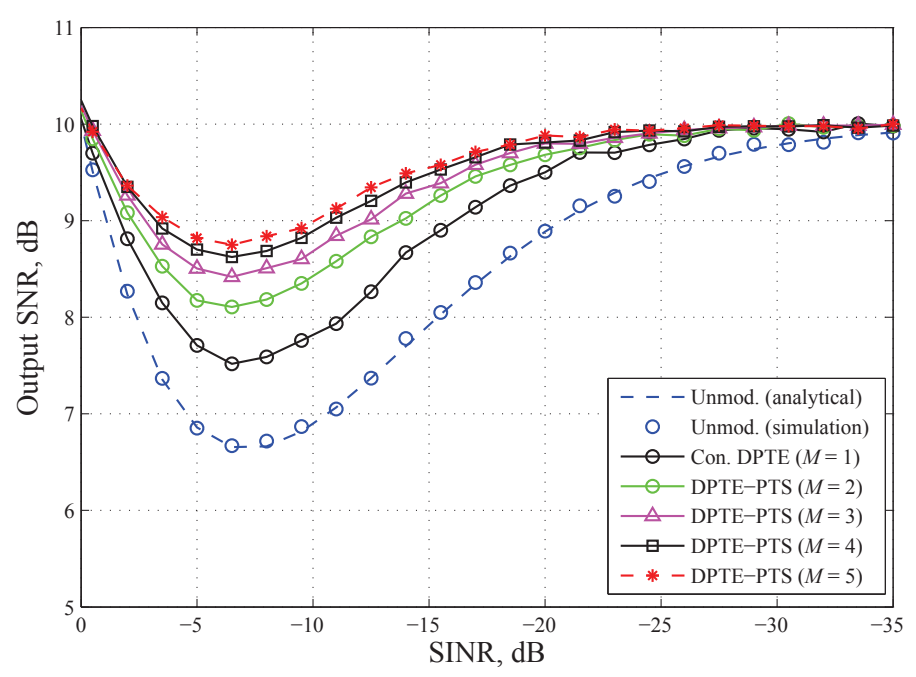

(b) $p=0.1$.

Figure 6: Output SNR versus SINR for the unmodified, conventional DPTE and DPTE-PTS methods for various values of $M$ when $p=0.01$ and 0.1 .

$$
P_{s}^{\text {unmod }}=\exp \left(-\frac{T^{2}}{2\left(\sigma_{s}^{2}+\sigma_{w}^{2}+\sigma_{i}^{2}\right)}\right) p
$$

\section{B. DPTE Method}

For the conventional DPTE and DPTE-PTS methods, $P_{s}$ is determined as

$$
P_{s}^{D P T E}=P\left(A_{r}>\mathrm{P} \mid \mathcal{H}_{1}\right) P\left(\mathcal{H}_{1}\right)
$$

Fig. 5 shows some numerical results of (17) along with simulation results for the unmodified, conventional DPTE and DPTE-PST systems. For the three systems it can be seen that $P_{s}$ improves as SINR becomes smaller and it approaches one for very low SINR values. This is due to the fact that in this region IN amplitudes are so high, compared to the useful OFDM signal, that all the three techniques can perfectly detect the noise pulses. At the other extreme, however, when IN is low $P_{s}$ is minimized. Similarly as in the previous section it is noticeable that as $M$ increases performance becomes better. In general, it can also be observed from Fig. 4 and 5 that $P_{m}$ and $P_{s}$ are inversely proportional.

\section{OUTPUT SNR PERFORMANCE}

For more quantitative characterization of the proposed method, we have conducted extensive computer simulations in this section to analyze the impact of DPTE-PTS method on the SNR at the output of the blanking device. The output SNR is determined as $\mathrm{SNR}_{D P T E}=\frac{\mathbb{E}\left[\left|\tilde{s}_{k}\right|^{2}\right]}{\mathbb{E}\left[\left|y_{k}-\tilde{s}_{k}\right|^{2}\right]}$.

Fig. 6 illustrates the output SNR versus SINR for the unmodified, conventional DPTE and DPTE-PTS techniques with different values of $M$ for $p=0.01$ and 0.1 . The analytical results of the unmodified method are obtained using 7 with the assumption of perfect estimation for IN characteristics. The good agreement between the analytical and simulation results indicates the accuracy of our simulation model. From this figure it is clearly seen that the proposed technique always outperforms both the unmodified and the conventional DPTE systems providing gains of up to $3.5 \mathrm{~dB}$ and $2 \mathrm{~dB}$ relative to the unmodified scheme when $M=5$ for $p=0.01$ and 0.1 , respectively. As anticipated, it is obvious that this enhancement increases as $M$ increases and becomes less significant when $M$ goes beyond 3. In addition, for both IN probabilities, it is noted that the proposed system yields about 1.5 dB SNR improvement compared to the conventional DPTE scheme at the same value of $M$.

\section{CONCLUSION}

In this paper we have evaluated the performance of DPTE method combined with a PAPR reduction technique, namely, PTS scheme, in OFDM-based PLC systems. The results clearly demonstrate the robustness and superiority of the proposed technique in terms of minimized probability of missed blanking, probability of blanking error and enhanced probability of successful detection. In addition, the output SNR was considered and it was shown that DPTE-PTS can provide up to $3.5 \mathrm{~dB}$ SNR gain with respect to the unmodified system and about $1.5 \mathrm{~dB}$ compared to the conventional DPTE technique. It was also found that increasing the number of PTS partitions will result in a better performance; this would be achieved at the expense of more computational complexity at the transmitter.

\section{REFERENCES}

[1] S. Galli, A. Scaglione, and Z. Wang, "For the grid and through the grid: The role of power line communications in the smart grid," Proc. IEEE, vol. 99, no. 6, pp. 998-1027, 2011.

[2] M. Ghosh, "Analysis of the effect of impulse noise on multicarrier and single carrier QAM systems," IEEE Trans. Commun., vol. 44, no. 2, pp. 145-147, Feb. 1996.

[3] S. V. Zhidkov, "Performance analysis and optimization of OFDM receiver with blanking nonlinearity in impulsive noise environment," IEEE Trans. Veh. Technol., vol. 55, no. 1, pp. 234-242, Jan. 2006.

[4] E. Del Re, R. Fantacci, S. Morosi, and R. Seravalle, "Comparison of CDMA and OFDM techniques for downstream power-line communications on low voltage grid," IEEE Trans. Power Del., vol. 18, no. 4, pp. 1104-1109, Oct. 2003. 
[5] J. Haring and A. J. H. Vinck, "Iterative decoding of codes over complex numbers for impulsive noise channels," IEEE Trans. Inf. Theory, vol. 49, no. 5, pp. 1251-1260, May 2003.

[6] O. P. H. et al., "Detection and removal of clipping in multicarrier receivers," Eur. patent appl. EP1043874, Oct. 2011.

[7] N. P. Cowley, A. Payne, and M. Dawkins, "COFDM tuner with impulse noise reduction," Eur. Patent Appl. EP1180851, Feb. 2002.

[8] S. V. Zhidkov, "On the analysis of OFDM receiver with blanking nonlinearity in impulsive noise channels," in Proc. Int. Symp. Intell. Signal Process. Commun. Syst., Nov. 2004, pp. 492-496.

[9] E. Alsusa and K. Rabie, "Dynamic peak-based threshold estimation method for mitigating impulsive noise in power-line communication systems," IEEE
Trans. Power Del., vol. 28, no. 4, pp. 2201-2208, Oct. 2013.

[10] K. Rabie and E. Alsusa, "Quantized peak based impulsive noise blanking in power-line communications," IEEE Trans. Power Del., Feb. 2014.

[11] S. H. Muller and J. B. Huber, "OFDM with reduced peak-to-average power ratio by optimum combination of partial transmit sequences," Elect. Lett., vol. 33, no. 5, pp. 368-69, Feb. 1997.

[12] R. van Nee and R. Prasad, "OFDM for wireless multimedia communications," Artech House, 2000.

[13] K. Rabie and E. Alsusa, "Preprocessing based impulsive noise reduction for power-line communications," to appear in IEEE Trans. Power Del., 2014. 